\title{
Profiling, Prediction, and Capping of Power Consumption in Consolidated Environments*
}

\author{
Jeonghwan Choi Sriram Govindan Bhuvan Urgaonkar Anand Sivasubramaniam \\ Department of Computer Science and Engineering \\ The Pennsylvania State University, University Park, PA, 16802 \\ \{jechoi,sgovinda,bhuvan,anand\}@cse.psu.edu
}

\begin{abstract}
Consolidation of workloads has emerged as a key mechanism to dampen the rapidly growing energy expenditure within enterprise-scale data centers. To gainfully utilize consolidation-based techniques, we must be able to characterize the power consumption of groups of co-located applications. Such characterization is crucial for effective prediction and enforcement of appropriate limits on power consumption-power budgets-within the data center. We identify two kinds of power budgets (i) an average budget to capture an upper bound on long-term energy consumption within that level and (ii) a sustained budget to capture any restrictions on sustained draw of current above a certain threshold. Using a simple measurement infrastructure, we derive power profiles-statistical descriptions of the power consumption of applications. Based on insights gained from detailed profiling of several applications-both individual and consolidated-we develop models for predicting average and sustained power consumption of consolidated applications. We conduct an experimental evaluation of our techniques on a Xen-based server that consolidates applications drawn from a diverse pool. For a variety of consolidation scenarios, We are able to predict average power consumption within $5 \%$ error margin and sustained power within 10\% error margin. Our sustained power prediction techniques allow us to predict close yet safe upper bounds on the sustained power consumption of consolidated applications.
\end{abstract}

\section{Introduction}

\subsection{Motivation}

To accommodate modern resource-intensive highperformance applications, large-scale data centers have grown at a rapid pace in a variety of domains ranging from research labs and academic groups to industry. The fast-growing power consumption of these platforms is a major concern due to its implications on the cost and

${ }^{*}$ In Proceedings of IEEE MASCOTS, Baltimore, MD, September 2008. This research was supported in part by the grant NSF CNS-0720456 and a gift from Cisco Systems, Inc. efficiency of these platforms as well as the well-being of our environment. Trends from such platforms suggest that the power consumption in data centers accounts for $1.2 \%$ of the overall electricity consumption in the U.S. More alarmingly, if current practices for the design and operation of these platforms continue, their power consumption is projected to keep growing at $18 \%$ every year. These observations have spurred great interest among providers of data centers to explore ways to dampen the growth rate of servers by doing better consolidation. For example, as workload conditions change, it may be desirable to pack hosted applications on to different subsets of racks/servers within the data center and turn off machines that are not needed $[5,6,20]$. Another major concern for data centers is the increase in power density of the servers which are reaching the limits of the power delivery and cooling infrastructure of these platforms, thereby raising reliability concerns. In response to this, existing research has attempted to reduce the peak power consumption both at the server level [11] as well as at the cluster level [22]. Consolidation further increases the power density of the servers, aggravating the reliability concerns of the facility.

Power budgets - upper bounds on power consumptionare a useful abstraction employed by several recently proposed techniques to address these energy and reliability related concerns in data centers $[11,21,22]$. Such power budgets need to be enforced at different levels of the spatial hierarchy. Previous work has looked at mechanisms to enforce power budgets both at the server level [17] and at the cluster level [31]. Typically power budgets are enforced either by throttling resource usage $[17,31]$ and/or migrating workloads $[18,27]$. In our opinion, these techniques often operate with inadequate information about the power consumption behavior of the hosted applications. As a result, when consolidating applications, such techniques could cause poor performance or less effective enforcement of power budgets. Solutions for consolidation in data centers have benefited from detailed studies of the resource needs of individual applications [30]. Insights gained from these studies have been utilized to build models for predicting the performance and resource usage behavior of consolidated applications [29]. Similar research on the power consumption of consolidated applications, however, has received much less attention. Such research would be useful to an energyfriendly operation and management of consolidated platforms in a variety of ways. First, it will facilitate the predic- 
tion and control of energy consumption in consolidated environments. Second, in combination with existing research on workload characterization and application modeling, it will facilitate meaningful trade-offs between energy costs and application performance. Third, it will enable data centers to operate in regimes that are profitable yet safe from power surges likely to be induced by aggressive consolidation. Finally, ongoing efforts to develop power benchmarks would also benefit from such characterization [25].

Two aspects of power consumption are of particular significance at all levels of the spatial hierarchy within a data center. First, the long-term average power consumption (several minutes to hours) within a sub-system dictates the energy costs involved in operating it. Second, the possibility of sustained power consumption above thresholds associated with fuses/circuit-breakers (typically a few seconds or even sub-second durations) critically affects the safe operation of devices protected by these elements. Thermal effects can also raise the need for both these budgets. At coarse spatial granularity (such as a room), average power may need to be curtailed to avoid excess heating. For smaller components (such as chips), power consumption must be controlled at finer time scales. In this paper, we characterize the power consumption of individual applications and use these characteristics to predict both the average and sustained power usage of consolidated applications.

Both average and sustained power consumption in a consolidated environment depend in non-trivial ways on the power consumption as well as resource usage patterns of the individual applications. Prediction of power consumption requires us to accurately identify these dependencies. Furthermore, the success of such prediction also depends on the methodology used to measure and characterize individual consumption. The design of measurement techniques and prediction models that can address these concerns is the focus of this paper.

\subsection{Research Contributions}

Using a simple combination of hardware and software tools, we design an offline technique to measure the power usage of individual applications. These power profiles are converted into convenient statistical descriptions of power usage. Similar profiles are obtained for resource usage of the applications. These profiles are then used to build predictive models for average and sustained power consumption of consolidated applications. Our profiling and prediction techniques are general enough to be useful for a wide variety of applications and consolidation scenarios.

We conduct an empirical evaluation of our techniques using a prototype server running the Xen virtual machine monitor [2]. This server is capable of consolidating multiple applications, each encapsulated within its own virtual machine. Our evaluation employs a wide variety of applications with diverse power and resource usage behavior to demonstrate the utility and general applicability of our models. Our offline profiling yields crucial insights into the power usage of applications and its relationship with their resource usage. Our predictive models, built upon these insights, appear promising. For a variety of consolidation scenarios, we are able to predict average power consumptions with in a $5 \%$ error-margin.

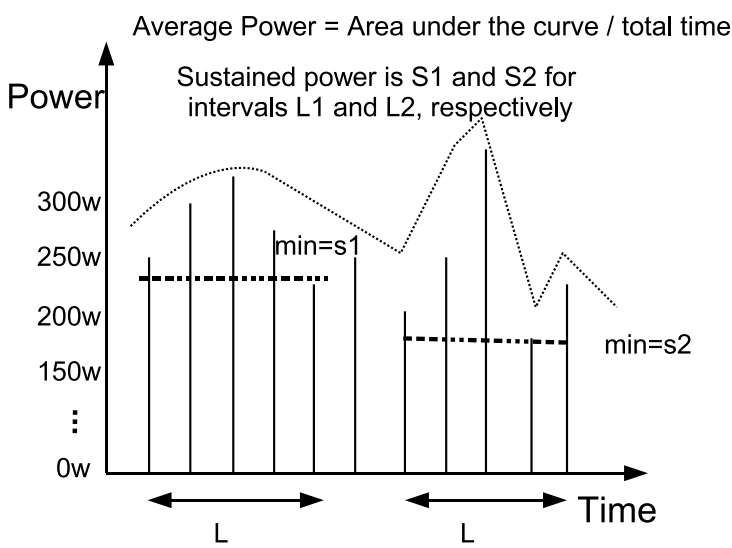

\section{Figure 1. Illustration of average and sustained power consumptions.}

\subsection{Road-map}

The rest of this paper is organized as follows. In Section 2, we provide necessary background on power consumption in data centers and formalize the notions of average and sustained power consumption. In Section 3, we develop an offline measurement technique for deriving power profiles of applications. In Sections 4 and 5, we develop and evaluate techniques for predicting average and sustained power consumption, respectively. We discuss related work in Section 6. Finally, we present concluding remarks in Section 7.

\section{Background}

\subsection{Power Consumption in Data Centers}

In a typical data center, a primary switch board distributes power among several Uninterrupted Power Supply Sub-stations (UPS; 1,000 KW) that, in turn, supply power to collections of Power Distribution Units (PDU; $200 \mathrm{KW}$.) A PDU is associated with a collection of server racks (up to 50.) Each rack has several chassis that host the individual servers. Power supply could be either at the server-level (as in rack-mounted systems) or at the chassis-level (as in blade servers.) At all these levels within the spatial hierarchy of a data center, fuses/circuit-breakers are used to protect equipment from surges in current drawn.

We focus on characterizing power consumption at the following levels: (a) at the lowest level, multiple applications are consolidated on a physical server, (b) at the higher levels, multiple servers are consolidated within a PDU.

\subsection{Average and Sustained Power Budgets}

Two aspects of the power consumption within each level of the spatial hierarchy described above play an important role in the safe and profitable operation of the data center. At time-scales over which consolidation decisions are made (which, in turn, may be related to the time-scales at 
which workload characteristics change), it may be desirable to limit the energy consumption within each level to values that yield acceptable trade-offs between application performance/revenue and energy costs. Such decision-making, likely to be done once every several minutes or hours (we will simply refer to time-scales of this order as long-term), might involve solving complex optimization problems to balance the performance/revenue yielded by operating a subset of the resources against the costs expended towards maintenance, operational power, and cooling. Regardless of the nuances of this decision-making, it necessitates mechanisms to enforce limits on the long-term energy expenditure within various levels. We refer to such a limit for a level as the $a v-$ erage power budget apportioned to it by the consolidation technique.

A second kind of budget, called the sustained power budget, results from the reliability needs of various hardware components in the data center defined by fuses ${ }^{1}$ or circuit breakers associated with that component. In literature, the phrase peak power is sometimes used for this aspect of power usage [11]. Sustained power budgets are defined over fairly small time periods - of the order of a few seconds or even milliseconds. A sustained power budget corresponds to a 2-tuple $(S, L)$, which specifies a bound on the maximum power $S$ that could be safely sustained over any interval of length $L$. This tuple is obtained from the time-current characteristics curve of a fuse. While a real time-current curve would correspond to multiple such tuples, for simplicity, we simply use a single tuple to represent the safety limits for a fuse/circuit-breaker. Figure 1 shows a hypothetical power series to illustrate the difference between average and sustained power. In this example, the average power consumption could be approximated by taking the average of the power samples in the entire time interval (energy/time). Note that the sustained power consumption for any interval of length $L$ corresponds to the minimum power consumed during that interval. This is because sustained power is the maximum power that was sustained throughout that interval. It is important to clearly understand this quantity to appreciate the difficulty of estimating it in consolidated settings that we explore in Section 5.

\section{Power Profiles: Measurement and Charac- terization}

In this section, we develop techniques to measure and characterize the power consumption of individual applications. Borrowing techniques from existing research, we also derive characterizations of their resource usage. Finally, we measure and characterize the power and resource usage consumption when these applications are consolidated. Taken together, these measurements set the background for techniques we develop in subsequent sections for predicting useful properties of power consumption in consolidated settings. Due to space constraints, we keep this section brief and invest more space on Sections 4 and 5.

\footnotetext{
${ }^{1} \mathrm{~A}$ fuse is a metal wire that melts when the current flowing through it exceeds certain thresholds, opening the underlying circuit and thereby protecting the circuit. Associated with a fuse is its time-current characteristic curve which shows the time required to melt the fuse for a given draw of current. A circuit breaker is similar to a fuse in its operation except that it could be reused after an episode of excessive current draw.
}

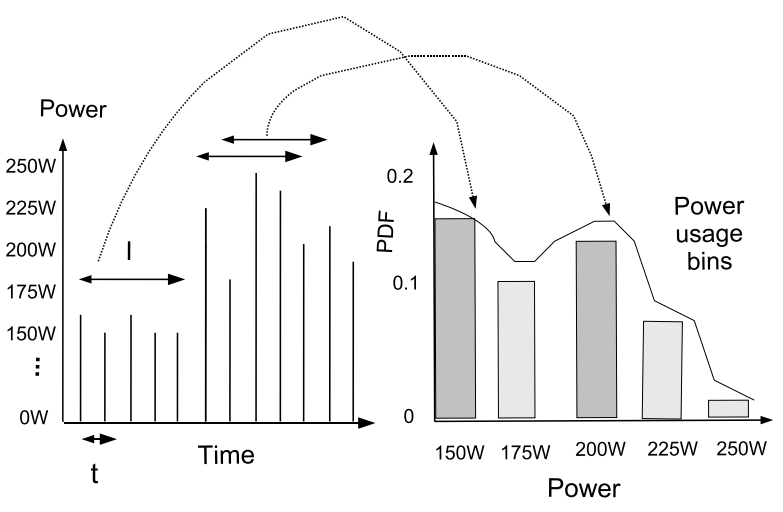

Figure 2. Illustration of the derivation of power usage distribution from a power profile for $t_{p}=$ $t$ and $I_{p}=I$.

\subsection{Empirical Derivation of Power and Resource Usage Profiles}

Our approach for characterizing the power and resource usage of an application employs an offline profiling technique similar to those existing in current research [30]. The profiling technique involves running the application on an isolated server. The application is then subjected to a realistic workload and a combination of hardware and software monitoring infrastructure is used to track its power and resource usage.

Profiling power consumption. We connect a multi-meter to the server used for our offline profiling and use it to measure the power consumption of the server once every $t_{p}$ time units. We refer to the resulting time-series of (instantaneous) power consumption samples as the power profile of the application. We find it useful to convert these power profiles into power usage distributions. Let $w_{A}^{I_{p}}$ be a random variable that represents the average power consumption of the application $A$ over durations of $I_{p}$ time units, where $I_{p}=k \cdot t_{p}$, ( $k$ is a positive integer.) Note that $w_{A}^{I_{p}}$ represents the average consumption over any consecutive interval of size $I_{p}$. It is estimated by shifting a time window of size $I_{p}$ over the power profile, and then constructing a distribution from these values. Figure 2 illustrates the process of converting a power profile into a power usage distribution. As part of our profiling, we also profile the idle power of the server running the applications (approx. 156W for our server).

Profiling resource usage. We use measurement techniques similar to those existing in research [30] to record resource scheduling events of interest. By recording CPU scheduling/de-scheduling instants for the virtual machine running our application, we derive its CPU usage profile, an ON-OFF time series of its CPU usage. Similarly, packet transmission/reception times and lengths yield its network bandwidth usage profile. We also record time series of memory consumption and disk I/O requests made by the application. Similar to power measurements, we find it useful to construct resource usage distributions from these pro- 
files. Finally, we also record application-specific performance metrics (e.g., response time, throughput.)

\subsection{Profiling Applications: Key Experimental Re- sults}

\begin{tabular}{|c|c|}
\hline \multicolumn{2}{|c|}{ Dell PowerEdge SC1450 Features [8] } \\
\hline \hline Processor & Two(2) Intel(R) Xeon 64bit 3.4 GHz \\
Main Memory & 2GB \\
Hard Disk(2) & WD Caviar 40GB 7200rpm \\
Network Interface & Dual embedded Intel Gigabit2 NICs \\
Power Supply & 450Wx1 \\
\hline
\end{tabular}

Table 1. Specifications of the server used for profiling.

\begin{tabular}{|c|c|}
\hline \multicolumn{2}{|c|}{ Signametrics SM2040 Features [23] } \\
\hline \hline Digits of Resolution & $6-1 / 2$ \\
Measurement Rates & $0.2 / \mathrm{sec}-1000 / \mathrm{sec}$ \\
Measurement Range (AC current) & $2.5 \mathrm{~A}$ \\
Interface & $\mathrm{PCI}$ \\
\hline
\end{tabular}

Table 2. Details of the multi-meter used in our profiling.

Our testbed consists of several Dell PowerEdge servers (details appear in Table 1.) We use one of these servers for running the applications that we profile. We connect a Signametrics SM2040 multi-meter (details appear in Table 2) in series with the power supply of this server. The multi-meter sits on the PCI bus of another server which is solely used for logging purposes. This multi-meter is capable of recording power consumption as frequently as once every millisecond.

The server running the application is connected to the multi-meter and we use the remaining servers to generate the workload for the application. In this paper, we report our observations for the representative applications listed in Table 3. In our environment, we observed that the total server power is proportional to the CPU utilization, so we find it useful to classify these applications based on their CPU usage. Applications in the SPEC CPU2000 suite are CPUsaturating, in that they are ready to use the CPU at all times. The remaining applications alternate between using the CPU and being blocked (e.g., on I/O, synchronization activities, etc.) and their CPU utilization depends on the workload they are offered. We profile these non-CPU-saturating applications at different workload intensities. TPC-W is profiled with the the number of simultaneous Web sessions varying from 10 to 100 , in increments of 10 . For experiments involving TPC-W, we represent the workload intensity as TPC-W(x) where " $\mathrm{x}$ " is the number of sessions. For experiments involving Streaming Media Server, 3Mbps is used as the streaming rate and we denote the workload as $\mathrm{SM}(\mathrm{x})$ where " $x$ " is the number of clients.

Throughout this section, we have $t_{p}=I_{p}=2 \mathrm{msec}$ ( $\mathrm{sam}$ pling interval.) We begin by observing the power distributions of our applications and comparing key properties. We present a subset of these results in Figure 3. Given that CPU consumes significantly more power than $\mathrm{I} / \mathrm{O}$ devices in our environment, not surprisingly, power distributions for non

\begin{tabular}{|c|c|}
\hline \multicolumn{2}{|c|}{ Applications } \\
\hline \hline TPC-W [28] & $\begin{array}{c}\text { 3-tiered NYU implementation of the TPC-W } \\
\text { transactional Web-based E-commerce benchmark }\end{array}$ \\
\hline Streaming Media & $\begin{array}{c}\text { Home-grown UDP streaming server, } \\
\text { runs with specified no. of clients and data rate }\end{array}$ \\
\hline SPEC CPU2000 [24] & SPEC CPU2000 suite (Art, Bzip2, Mcf, Mesa) \\
\hline
\end{tabular}

Table 3. Salient properties of our applications. TPC-W and Streaming-server are non CPUsaturating, whereas applications in the SPEC CPU2000 suite are CPU-saturating.

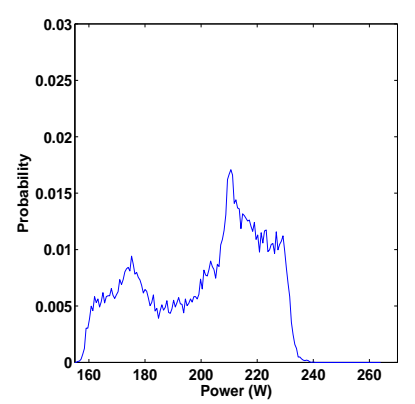

(a) Streaming, 60 clients $(\mathrm{SM}(60))$

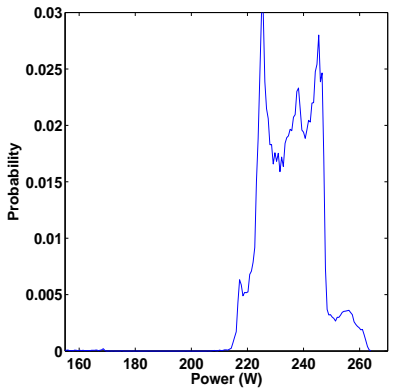

(b) $\mathrm{Mcf}$

\section{Figure 3. Power distributions of Streaming server and Mcf compared.}

CPU-saturating application (Figures 3 (a)) are found to exhibit higher variance than CPU-saturating application (Figures 3 (b).) This observation leads us to realize that one primary contributor to variance in the power usage of an application is a change in its CPU scheduling state. The CPU profile of a non CPU-saturating application exhibits a ON-OFF behavior, corresponding to the application being in running and blocked states, respectively. When such an application blocks, its power consumption corresponds to the server's idle power. This ON-OFF CPU usage contributes to the higher variance in its power consumption.

\section{Average Power Prediction}

In this section, we develop techniques to predict the average power consumption of a server which consolidates multiple applications. The inputs to any of our prediction algorithms are the power and resource usage distributions of the individual applications. We conduct experiments to evaluate the efficacy of our techniques.

\subsection{Baseline Prediction}

We begin with the following simple baseline predictor for average power consumption of a server on which $n$ applications $A_{1}, \cdots, A_{n}$ are consolidated. Our predictor employs a sum of the average power consumptions of the individual applications, weighted by their respective CPU utilizations,

$$
\bar{P}_{A_{1}, \cdots, A_{n}}=\sum_{i=1}^{n}\left(\bar{P}_{A_{i}} \cdot R_{A_{i}}^{c p u}\right)+\bar{P}^{i d l e} \cdot\left(1-\sum_{i=1}^{n} R_{A_{i}}^{c p u}\right)
$$


where $\bar{P}_{A_{i}}$ is the average of the power distribution of the application $A_{i}$ (obtained from the offline profile); and $R_{A_{i}}^{c p u}$ $\left(0 \leq R_{A_{i}}^{c p u} \leq 1\right)$ is the CPU allocation for it. ${ }^{2}$ Note that $\bar{P}_{A_{i}}$ is the average of the total system power measured when application $A_{i}$ alone is running on the server and this includes the power consumed by the applications in all the components of the server. The first term captures the power dissipation of the server when the CPU is busy, whereas the second term is for when it is idle.

We present in Table 4 the efficacy of baseline prediction in three consolidation settings, each co-locating a pair of applications.

\begin{tabular}{|c|c|c|c|}
\hline $\begin{array}{c}\text { Applications } \\
\text { consolidated }\end{array}$ & $\begin{array}{c}\text { Baseline prediction } \\
(\mathrm{W})\end{array}$ & $\begin{array}{c}\text { Observed } \\
\text { average }(\mathrm{W})\end{array}$ & $\begin{array}{c}\text { Error } \\
(\%)\end{array}$ \\
\hline Art + Mesa & 222.1 & 226.1 & 1.7 \\
Art + TPC-W(60) & 209.3 & 224.6 & 6.8 \\
TPC-W(10)+ TPC-W(60) & 167.4 & 190.1 & 11.9 \\
\hline
\end{tabular}

\section{Table 4. Baseline predictor of average power consumption.}

In the first consolidation setting, two CPU-saturating applications Art and Mesa, time-share the CPU equally, and our baseline approach proves to be an excellent predictor of average power. In the latter two consolidation settings, where we have non-CPU-saturating applications, we observe increasing error margins in the baseline prediction. The main reason for these inaccuracies is that the first quantity in Eq. 1, $\bar{P}_{A_{i}}$ represents the average of the entire power distribution for application $A_{i}$ including the durations when the CPU was idle. Non-CPU-saturating applications can have significant such idle periods. Upon consolidation, however, these idle durations are likely to be occupied by other co-located applications. Therefore, we need to employ the average power consumption by the application only over durations when it was using the CPU.

\subsection{Improving Prediction of Average Power}

We are interested in determining the average power consumed by an application only over durations when it was scheduled to run on the CPU. This can be estimated by considering the power distribution beyond its $100 \cdot\left(1-U_{A}^{c p u}\right)^{t h}$ percentile, where $U_{A}^{c p u}$ is the average CPU usage for $A$ as obtained from its offline CPU usage profile (note that $U_{A}^{c p u}$ should not be confused with the allocation $R_{A}^{c p u}$ that we encountered above.) The average power for this subset of the entire distribution corresponds exactly to the power consumed when the application was running on the CPU. Figure 4 shows this for TPC-W(60) whose CPU utilization was $40 \%$. We denote this quantity by $P_{A_{i}}^{\text {busy }}$ and replace $P_{A_{i}}$ in Eq. 1 with it. In this example, $P_{t p c w}^{\text {busy }}$ was found to be $225 \mathrm{~W}$, while $P_{t p c w}$ was $185 \mathrm{~W}$ - note the difference.

Based on observations from several consolidation scenarios, we find that this enhancement results in improved predictions of average power. As specific examples, recall the

\footnotetext{
${ }^{2}$ These CPU allocations for the applications are set by the administrator in the consolidated setting. Figuring out these allocations for the applications is done using well-studied techniques for application modeling [29].
}

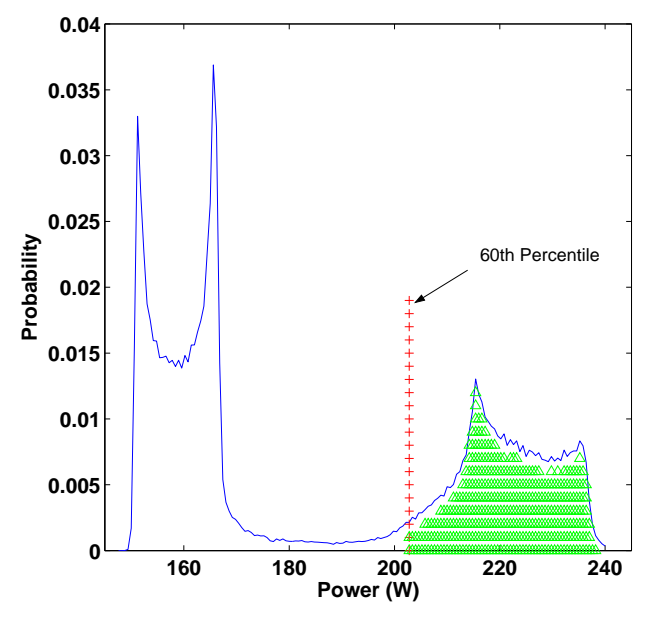

\section{Figure 4. Capturing the non-idle power portion for TPC-W(60).}

prediction of average power for a server hosting (a) Art and TPC-W(60) and (b) TPC-W(60) and TPC-W(10) (Table 4.) With this enhancement, our estimates of average power improved from $209 \mathrm{~W}$ to $226 \mathrm{~W}$ for (a), and from $167 \mathrm{~W}$ to $188 \mathrm{~W}$ for (b) which reduces the error margins to $1.76 \%$ and $1.3 \%$, respectively. In Figure 5, we present the performance of our baseline and enhanced predictors for a variety of consolidation scenarios. Our enhanced technique is able to predict within $5 \%$ of the observed power while the baseline approach has up to $20 \%$ error margin.

Our prediction technique assumes that the total system power is in proportion with the CPU utilization of the application. Though this turns out to be a good assumption for our environment (where CPU is the dominant consumer of power), this may not be the case where more power consuming I/O components (e.g., a graphics card, networking equipment, etc.) are deployed.

\section{Sustained Power Prediction}

Next, we turn our attention to sustained power budgets in consolidated servers. Recall that sustained power budgets are enforced at different levels in a data center to ensure that the applications consolidated under that level do not cause the capacity of the corresponding PDU to be exceeded. In Section 5.1 we develop mechanisms to predict the sustained power consumption of applications consolidated on a server. At a single server level, sustained power prediction boils down to finding the possibility of a single server consuming enough power to reach the limit of its power supply. Though this may seem unlikely given the fact that the capacity of a server-level power supply is typically much higher than its peak power consumption [10], this method will later be extended to predict the possibility of applications consolidated on a set of servers reaching the capacity of their PDU which is very much a concern. Also, taking a cue from the aforementioned over-provisioning present in existing power supplies, recent research [17] has suggested using lower capacity (and cheaper) power supplies for servers to cut costs. Such an approach could make use of effective pre- 


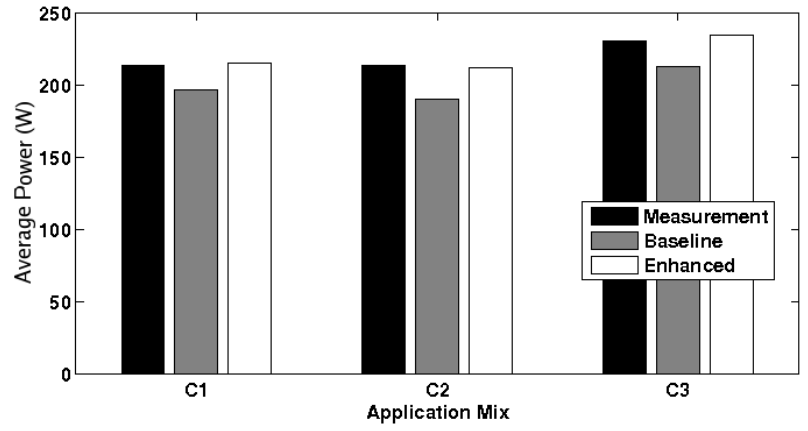

Figure 5. Comparison of our predictors for a variety of consolidation scenarios. In C1 and $\mathrm{C2}$, the applications are consolidated on the same physical processor and the second processor is idle. In C3, two applications each are consolidated on each processor. C1: TPC-W(60)+Bzip2+SM(100) C2: TPC-W(60)+Bzip2+SM(100)+SM(100) C3: Processor1: TPC-W(60)+SM(100), Processor2: SM(100)+Bzip2.

diction techniques to determine the possibility of the limit of a power supply being violated. In Section 5.2 we extend our approach to predict sustained power violation at the PDU or rack level (across a set of servers.)

\subsection{Sustained Power Prediction for a Server}

Recall that our goal is to predict the probability $\operatorname{Pr}_{A_{1}, \cdots, A_{n}}(S, L)$, upon consolidating $n$ applications $A_{1}, \cdots, A_{n}$ on a server, of $S$ or more units of power being consumed for any $L$ consecutive time units. We will refer to $\mathrm{Pr}_{-}$as the probability of violating the sustained power budget. Note the trivial feasibility requirement on the sustained power budget that it always be higher than the idle power-if this does not hold, the server would be inoperable. I/O power contribution for our set of applications and servers is very low and, therefore, we do not consider it for predicting sustained power. Note that we are not ignoring power contributed by I/O components. The entire server power (from all the components in the server) is assumed to be proportional to the CPU utilization.

5.1.1. Baseline Prediction To bring out the difficulties in predicting the probability of a given sustained power consumption, we evaluate a simple baseline approach that operates as follows. It first estimates the number of slots $m_{i}$, each of length $t_{p}$ (recall that $t_{p}$ is our power measurement granularity), during which the application $A_{i}$ is expected to occupy the CPU over a duration of $L$ time units,

$$
m_{i}=\frac{L \cdot R_{A_{i}}^{c p u}}{t_{p}}
$$

where $R_{A_{i}}^{c p u}$ is the CPU allocation for application $A_{i}$ in the consolidated setting. Assuming stationarity of power con- sumption across durations of length $t_{p}$ for each of the applications, the probability of violation is estimated as,

$$
\operatorname{Pr}_{A_{1}, \cdots, A_{n}}(S, L)=\prod_{i=1}^{n}\left\{\operatorname{Pr}\left(w_{A_{i}}^{t_{p}} \geq S\right)\right\}^{m_{i}}
$$

where $\operatorname{Pr}\left(w_{A_{i}}^{t_{p}} \geq S\right)$ is the probability that application $A_{i}$ 's power consumption obtained from its power distribution at the granularity of $t_{p}$ time units exceeds the sustained-power limit, $S$. Note that the above assumes independence among the execution patterns of co-located applications-a reasonable assumption in our settings. We make this assumption throughout this section. There are three key shortcomings in the baseline approach.

Shortcoming (A) due to assuming saturated CPU First, the baseline approach does not capture the likelihood that the CPU could be idle for some portion of given durations of length $L$. Any such duration should not qualify as one where a violation of sustained power budget occurs (recall we assume that the sustained budget is greater than idle power.)

Shortcoming (B) due to stationarity assumption Second, the assumption of stationarity of power consumption at the granularity of $t_{p}$ time units holds only for a very selected type of applications. Among our set of applications, this applies only to some of the CPU-saturating applications which do not exhibit large temporal variation in their power consumption. An example of an application that does exhibit such variations is the CPU-saturating Mcf-it exhibits temporal variations in its memory access patterns resulting in variations in its power consumption measured at the granularity of $t_{p}$ units. We have already seen that all our nonCPU-saturating applications exhibit significant variations in power usage.

Shortcoming (C) due to ignoring CPU usage variation Finally, the baseline approach assumes that the CPU usage of each of the co-located applications would be precisely equal to their CPU allocations $\left(R_{A_{i}}^{c p u}\right.$ for application $\left.A_{i}\right)$ over any period of length $L$. Again, while this assumption is fine for a set of co-located CPU-saturating applications whose CPU usage patterns do not exhibit variability, it introduces inaccuracies when there is even one application that does not adhere to such behavior. In particular, it becomes inaccurate when predicting the sustained power behavior of a set consisting of one or more non-CPU-saturating applications (when these applications are blocked on I/O activities, those idle periods will likely be used by other co-located applications resulting in a different CPU allocation than specified by $R_{A_{i}}^{c p u}$ for the applications.)

In the rest of this section, we will address these three shortcomings.

\subsubsection{Improving Prediction of Sustained Power}

Addressing shortcoming (A). We had encountered a similar problem when dealing with reduction of average power. The idle periods included in the CPU profiles of non-CPUsaturating applications must be handled correctly because 
upon consolidation they are likely to be occupied by other co-located applications. Exactly as in Section 4.2, we remove this idle portion by only considering the power distribution beyond its $100\left(1-U_{A}^{c p u}\right)^{t h}$ percentile, where $U_{A}^{c p u}$ is the average CPU usage for $A$ as obtained from its CPU usage profile.

Addressing shortcoming (B). This problem arose because we used the distribution of power over $t_{p}$ units to predict the probability that an application $A_{i}$ consumes power above $S$ units for $T_{m_{i}}=m_{i} \cdot t_{p}$ consecutive time units during which it occupies the CPU. We can improve this prediction by using the power profile for $A_{i}$ to explicitly find this probability, rather than relying on the power distribution over $t_{p}$ time units. Equivalently, given $m_{i}$, we are interested in the power distribution over $m_{i} \cdot t_{p}$ time units. This distribution is easily derived from the power profile by moving a window the size of $m_{i} \cdot t_{p}$ time units, shifting it by $t_{p}$ units. For each such window, we find the maximum sustained power throughout that duration by taking the minimum power sample within that window. The samples obtained from these windows are converted into a distribution of sustained power over $m_{i} \cdot t_{p}$ time units. With this modification, our predictor takes the following form (compare with Eq. 2),

$$
\operatorname{Pr}_{A_{1}, \cdots, A_{n}}(S, L)=\prod_{i=1}^{n}\left\{\operatorname{Pr}\left(w_{A_{i}}^{T_{m_{i}}} \geq S\right)\right\}
$$

We provide an example to illustrate the seriousness of this problem. For TPC-W, the probability of violating a budget of $(200 \mathrm{~W}, 2 \mathrm{~ms})$ as obtained from TPC-W power profile collected at $2 \mathrm{~ms}$ is $99.64 \%$. The probability of violating 200W for a period of 1 second would be approximated as $(.9964)^{500}$ which is $16.47 \%$. But when we compute the actual sustained power by moving window of size $1 \mathrm{sec}$ over TPC-W time series, we find this probability to be $21.55 \%$.

Addressing shortcoming (C). We find this to be the most interesting and challenging shortcoming to address. Recall that this shortcoming arose because we assumed that the applications would always consume the CPU exactly in accordance with their their CPU allocations $\left(R_{A_{i}}^{c p u}\right.$ for application $A_{i}$ ). We attempt to alleviate this problem by incorporating into our prediction the multiple ways in which the CPU could be shared among the applications over durations of length $L$. We derive this using the individual applications' CPU profiles collected over duration of length $L$. Let $\operatorname{Pr}\left\{U_{\left(A_{1}, \cdots, A_{n}\right)}^{L}=\left(c_{1}, \cdots, c_{n}\right)\right\}$ be the probability that $\left(c_{1}, \cdots, c_{n}\right)$, are the fractional CPU consumptions of applications $\left(A_{1}, \cdots, A_{n}\right)$, respectively, over all intervals of length $L$ in our consolidated setting. We estimate this for all possible combinations of $\left(c_{1}, \cdots, c_{n}\right)$ as follows. The CPU utilization of the applications in the consolidated setting depends mainly on two things: (a) the CPU reservation of applications in the consolidated setting and (b) the CPU requirement of the applications (both over periods of length $L$ ). When the reservation is higher than the requirement, then it means the application has spare CPU which could be used by other applications whose reservations are smaller than their requirements. Most reservation-based schedulers (as

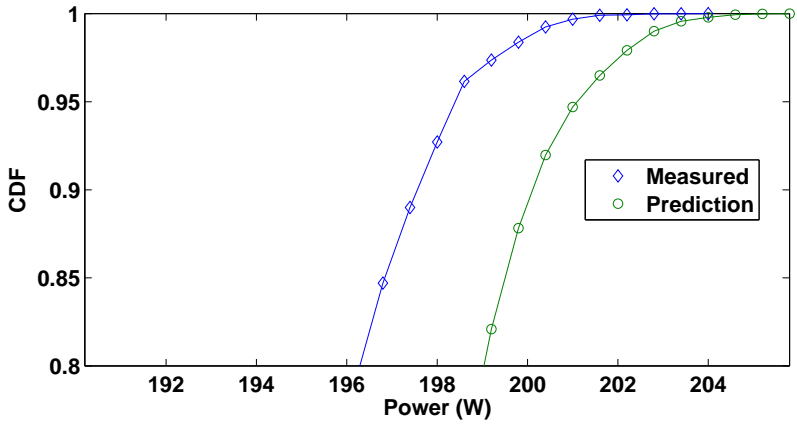

Figure 6. Comparison of measured and pre-
dicted sustained power consumption ( $=1$
sec) for a server consolidating TPC-W(60),
TPC-W(60) and Bzip2 with reservations $60 \%$,
$20 \%$ and, $20 \%$, respectively.

ours) divides this spare CPU equally among these needy applications. Our estimate for the CPU utilization of applications in the consolidated setting takes the above into account. We start by constructing the distributions of fractional CPU requirements for every application over durations of length $L$. These distributions can be easily derived from the CPU usage profiles of the applications. Let $\operatorname{Pr}\left(U_{A_{i}}^{L} \geq c\right)$ represent the probability that the CPU utilization of application $A_{i}$ over duration of length $L$ exceeds $c(0 \leq c \leq 1)$. We construct CPU requirement bins (RB) for every application over bins of length $\delta, R B_{A_{i}}[\delta, 2 \delta, 3 \delta, \cdots,(1-\delta), 1]$, where $R B_{A_{i}}[j]=\operatorname{Pr}\left(U_{A_{i}}^{L} \geq(j-\delta)\right)-\operatorname{Pr}\left(U_{A_{i}}^{L} \geq j\right)$, that is each bin represents the probability of utilization being within its bin boundaries.

For simplicity, we conduct the rest of the discussion in the context of two applications, $A_{1}$ and $A_{2}$. We are interested in finding $\operatorname{Pr}\left(U_{\left(A_{1}, A_{2}\right)}^{L}=\left(c_{1}, c_{2}\right)\right)$ (which is the probability that CPU utilization of application $A_{1}$ is $c_{1}$ and that of $A_{2}$ is $c_{2}$ ) for $c_{1}=\delta, 2 \delta, \cdots 1$ and $c_{2}=\delta, 2 \delta, \cdots 1$. It could be obtained by,

$$
R B_{A_{1}}[\delta, \cdots, 1] \cdot\left(R B_{A_{2}}[\delta, \cdots, 1]\right)^{\prime}
$$

where $\left(R B_{A_{2}}[\delta, \cdots, 1]\right)^{\prime}$ represents the transpose of the array $R B_{A_{2}}[\delta, \cdots, 1]$. Multiplying these two one dimensional matrices will generate a two dimensional matrix which provides the probability for all utilization pairs $\left(c_{1}, c_{2}\right) .{ }^{3}$ Note that in $\operatorname{Pr}\left\{U_{\left(A_{1}, A_{2}\right)}^{L}=\left(c_{1}, c_{2}\right)\right\},\left(c_{1}+c_{2}\right)$ ranges from 0 to 2 . But in a consolidated setting, utilization of the CPU cannot go beyond $100 \%$. Let $r_{1}$ and $r_{2}$ be the reservations for the application $A_{1}$ and $A_{2}$ respectively. We estimate the utilization of the applications in the consolidated setting as follows,

$$
\begin{aligned}
& \text { 1: for all }\left(c_{1}, c_{2}\right) \text { such that } c_{1}+c_{2}>1 \text { do } \\
& \text { 2: } \quad \text { if }\left(c_{1}>r_{1}\right) \text { and }\left(c_{2}>r_{2}\right) \text { then } \\
& \text { 3: } \quad \operatorname{Pr}\left(U_{\left(A_{1}, A_{2}\right)}^{L}=\left(r_{1}, r_{2}\right)\right)=\operatorname{Pr}\left(U_{\left(A_{1}, A_{2}\right)}^{L}=\left(r_{1}, r_{2}\right)\right)+ \\
& \quad \operatorname{Pr}\left(U_{\left(A_{1}, A_{2}\right)}^{L}=\left(c_{1}, c_{2}\right)\right) \\
& \text { 4: } \quad \text { else if }\left(c_{1}>r_{1}\right) \text { and }\left(c_{2}<r_{2}\right) \text { then }
\end{aligned}
$$

\footnotetext{
${ }^{3}$ This extends easily to $n$ applications; we omit these details here.
} 


$$
\begin{array}{ll}
\text { 5: } & \quad \operatorname{Pr}\left(U_{\left(A_{1}, A_{2}\right)}^{L}=\left(1-c_{2}, c 2\right)\right)=\operatorname{Pr}\left(U_{\left(A_{1}, A_{2}\right)}^{L}=(1-\right. \\
& \left.\left.\quad c_{2}, c 2\right)\right)+\operatorname{Pr}\left(U_{\left(A_{1}, A_{2}\right)}^{L}=\left(c_{1}, c_{2}\right)\right) \\
\text { 6: } & \text { else if }\left(c_{1}<r_{1}\right) \text { and }\left(c_{2}>r_{2}\right) \text { then } \\
\text { 7: } & \operatorname{Pr}\left(U_{\left(A_{1}, A_{2}\right)}^{L}=\left(c 1,1-c_{1}\right)\right)=\operatorname{Pr}\left(U_{\left(A_{1}, A_{2}\right)}^{L}=(c 1,1-\right. \\
& \left.\left.\quad c_{1}\right)\right)+\operatorname{Pr}\left(U_{\left(A_{1}, A_{2}\right)}^{L}=\left(c_{1}, c_{2}\right)\right) \\
\text { 8: } & \text { end if } \\
\text { 9: } & \text { end for }
\end{array}
$$

Lines 2 and 3 handles the case when the (fractional) CPU requirement of both the application is above their reservations, in this case the CPU usages of the applications in the consolidated setting would be $\left(r_{1}, r_{2}\right)$. Lines 4 through 7 handles the case when the CPU requirement of one application is below its reservation and that of the other application is above its reservation, in which case, the needy application gets CPU from the surplus of the other. Also notice that for all durations with some idle portion $\left(c_{1}+c_{2}<1\right)$, the probability of sustained power violation would be zero (recall the trivial feasibility requirement for sustained power budget which requires it to be higher than idle power.) This is captured by line 1 of the algorithm.

For any duration of length $L$ units where the applications $A_{1}$ and $A_{2}$ occupy the CPU for $c_{1}$ and $c_{2}$ time units, the probability of sustained power ( $\mathrm{S}$ watts) violation is given by, $\operatorname{Pr}_{A_{1}, A_{2}}(S, L)=\operatorname{Pr}\left(w_{A_{1}}^{c_{1}} \geq S\right) * \operatorname{Pr}\left(w_{A_{2}}^{c_{2}} \geq S\right)$ (similar to what was described in Eq. 3.) We use the above equation to find the probability of sustained power violation for all possible $\left(c_{1}, c_{2}\right)$ pairs as follows,

$$
\begin{aligned}
& \text { 1: } \operatorname{Pr}_{A_{1}, A_{2}}(S, L)=0 \\
& \text { 2: for } i=0 \text { to } 1 ; j=1 \text { to } 0 ; i=i+\delta ; j=j-\delta \text { do } \\
& \text { 3: } \quad \operatorname{Pr}_{A_{1}, A_{2}}(S, L)=\operatorname{Pr}_{A_{1}, A_{2}}(S, L)+\operatorname{Pr}\left(w_{A_{1}}^{i} \geq\right. \\
& \quad S) \cdot \operatorname{Pr}\left(w_{A_{2}}^{j} \geq S\right) \cdot \operatorname{Pr}\left\{U_{\left(A_{1}, A_{2}\right)}^{L}=(i, j)\right\}
\end{aligned}
$$$$
\text { 4: end for }
$$

Line 2 of the above algorithm loops through all possible $\left(c_{1}, c_{2}\right)$ pairs that add up to 1 (possible violation regions). The algorithm predicts the probability of sustained power budget violation for a given value of the power budget $S$. We run the above algorithm for $L=1 \mathrm{sec}$ and varying $S$ from 0 to $300 \mathrm{~W}$ for our experiments. Figure 6 and Table 5 evaluate our prediction mechanism for a server consolidating 3 applications. We are able to bound the tail of the sustained power consumption within $2 \%$ error margin (Table 5).

\begin{tabular}{|c|c|c|c|}
\hline $\begin{array}{c}\text { Probability of } \\
\text { violation }\end{array}$ & $\begin{array}{c}\text { Measured } \\
\text { Sustained power }(\mathrm{W})\end{array}$ & $\begin{array}{c}\text { Predicted } \\
\text { sustained power }(\mathrm{W})\end{array}$ & $\begin{array}{c}\text { Error } \\
(\%)\end{array}$ \\
\hline $20 \%$ & 196.3 & 199.0 & 1.37 \\
$10 \%$ & 197.5 & 200.1 & 1.31 \\
$1 \%$ & 200.2 & 202.8 & 1.29 \\
$0 \%$ & 204.0 & 207.0 & 1.47 \\
\hline
\end{tabular}

Table 5. Efficacy of sustained power prediction on a server consolidating TPC-W(60), TPCW(60) and Bzip2 with reservations $60 \%, 20 \%$, and $20 \%$, respectively.

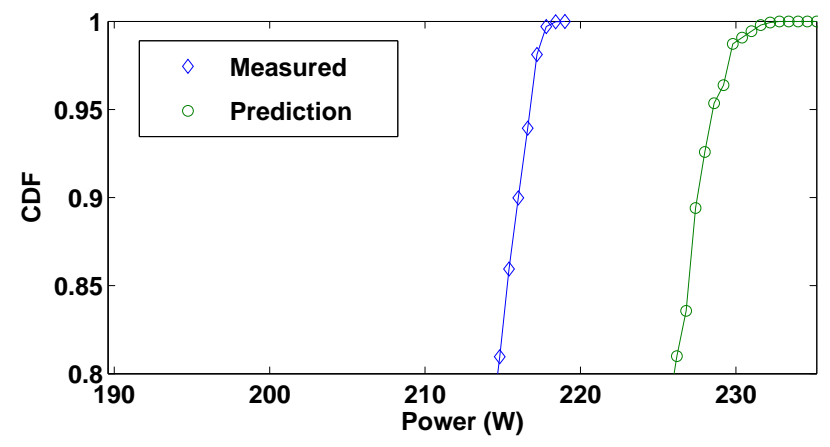

Figure 7. Comparison of measured and sustained power consumption ( $L=1 \mathrm{sec})$ of a PDU connected to server1 (running TPC-W(60) and $\mathrm{SM}(100)$ ) and server2 (running $\mathrm{SM}(100)$ and Bzip2).

\begin{tabular}{|c|c|c|c|}
\hline $\begin{array}{c}\text { Probability of } \\
\text { violation }\end{array}$ & $\begin{array}{c}\text { Measured } \\
\text { Sustained power (W) }\end{array}$ & $\begin{array}{c}\text { Predicted } \\
\text { sustained power }(\mathrm{W})\end{array}$ & $\begin{array}{c}\text { Error } \\
(\%)\end{array}$ \\
\hline $20 \%$ & 214.0 & 226.2 & 5.70 \\
$10 \%$ & 215.5 & 227.6 & 5.61 \\
$1 \%$ & 217.5 & 230.4 & 5.93 \\
$0 \%$ & 219.0 & 235.2 & 7.39 \\
\hline
\end{tabular}

Table 6. Efficacy of sustained power prediction on a PDU consolidating server1 (running TPC-W(60) and SM(100)) and server2 (running SM(100) and Bzip2).

\subsection{Sustained Power Prediction Across Multiple Servers}

Having predicted the sustained power consumption of a single server, we next predict the probability of sustained power budget violation across a set of servers. Our goal is to predict the probability $\operatorname{Pr}_{B_{1}, \cdots, B_{m}}(S, L)$, (that upon consolidating $m$ servers $B_{1}, \cdots, B_{m}$ on a PDU) of $S$ or more units of power being consumed by the PDU for any $L$ consecutive time units. Unlike the case when applications time share the server, in this case, the applications are running simultaneously and therefore the power consumption would add up. Recall from section 2.2 that we are interested in finding the minimum power consumption of the PDU over periods on length $L$ time units. This minimum power consumption of the PDU (consisting of set of servers) is upper bounded by the sum of average power (over intervals of length $L$ time units) of the individual servers. The proof is very simple, Consider two sets $U$ and $V$ consisting of $k$ elements each. Let $W$ be a set obtained by adding any permutation of the set $U$ with any permutation of the set $V$ (Note that set $W$ also has $k$ elements). The minimum value in set $W, W_{\text {min }}$ is upper bounded by its average, $W_{\text {avg }}\left(W_{\min } \leq W_{\text {avg }}\right)$. Note that average of the set $W$ is nothing but the sum of the averages of the sets $U$ and $V$. ( $\left.W_{a v g}=U_{a v g}+V_{a v g}\right)$ Therefore the sum of the averages of the sets $U$ and $V$ forms the upper bound of the minimum in set $W\left(W_{\min } \leq U_{a v g}+V_{a v g}\right)$.

We use the above idea to bound the maximum power sustained by the PDU. This can be achieved in 2 steps:

(Step1) Find the distribution of average power consumption 
of individual servers (connected to the PDU) over intervals of length $L$ time units.

(Step2) Add all these average power distributions. Assuming individual consumptions to be independent $-\mathrm{a}$ reasonable assumption - the resulting distribution of the aggregate can be computed from elementary probability theory. ${ }^{4}$

Step1 can be easily achieved by slightly modifying the techniques developed in section 5.1. Initially we estimate the CPU utilization of the consolidated applications, $\operatorname{Pr}\left(U_{\left(A_{1}, \cdots, A_{n}\right)}^{L}=\left(c_{1}, \cdots, c_{n}\right)\right)$ for all $\left(c_{1}, \cdots, c_{n}\right)$ and then instead of finding the minimum power consumption of the server, we compute the average power consumption of the server using the distribution of average power consumption of the individual applications (obtained from offline profiling) over intervals of length $L$ time units. Step2 is straightforward.

Figure 7 and Table 6 evaluates our prediction mechanism for a server consisting of 2 processors each consolidating 2 applications. Since we do not have an infrastructure that could measure power consumption of a set of servers, we did our evaluation on a single server with 2 processors. This is analogous to an environment with two servers connected to a PDU. Note from Table 6 that we are able to bound the tail of the sustained power with reasonable accuracy (within $8 \%$ ). Even though our approach provides an upper bound for the sustained power consumption, it is not a tight upper bound. As part of our future work, we intend to develop mechanisms that provide a much tighter upper bound on the sustained power consumption.

\section{Related Work}

While limitation on battery lifetime has been the main concern for mobile [13] and embedded systems, servers [4] have mainly been focusing on reducing energy consumption and handling reliability constraints imposed due to electrical and cooling limits.

Reducing energy consumption. The tremendous increase in power consumption over the last few years in mainly attributable to the growth in the number of servers, with only a small percentage associated with increase in the power use per unit. In an attempt to reduce the number of active servers, mechanisms to dynamically turn servers on/off based on utilization have been explored $[5,6,20]$. While the above research looked at reducing the number of servers, $\mathrm{Fe}-$ mal et al. suggested that over-provisioning servers may increase the performance of throughput-oriented applications without compromising on the power budget of the infrastructure [12]. The inter-play of power and performance for both uni-processors and multi-processors has been studied in great detail $[1,3,26]$. Chase et al. considered energyaware resource provisioning in a data center in accordance with negotiated QoS agreements [6]. Merkel et al. [18] did

\footnotetext{
${ }^{4}$ This is done using the $\mathrm{z}$-transform. The z-transform of a random variable $U$ is the polynomial $Z(U)=a_{0}+z a_{1}+z^{2} a_{2}+\cdots$ where the coefficient of the $i^{t h}$ term represents the probability that the random variable equals $i$ (i.e., $U(i)$ ). If $U_{1}, U_{2}, \ldots, U_{k+1}$ are $k+1$ independent random variables, and $Y=\sum_{i=1}^{k+1} U_{i}$, then $Z(Y)=\prod_{i=1}^{k+1} Z\left(U_{i}\right)$. The distribution of $Y$ can then be computed using a polynomial multiplication of the z-transforms of $U_{1}, U_{2}, \cdots, U_{k+1}$.
}

accounting and capping of energy consumption for virtual machines consolidated on a single server. Nathuji et al. [19] looked at extending power management capabilities for virtual machines. We believe that the above techniques for energy management will greatly benefit from our average power prediction both in deciding the energy budgets for the servers as well as the placement of applications.

Reliability concerns. Felter et al. proposed a technique that reduces the peak power demand on a server by dynamically distributing the power among the system components based on their requirement [11]. Lefurgy et al. recently presented a technique that uses system-level power measurement to cap the peak power consumption of the server while maintaining the system at the highest possible performance state [17]. Wang et al. devised a power capping mechanism for a cluster of servers [31]. Ranganathan et al. and Fan et al. did extensive profiling of real-world server clusters and both observed that the probability of synchronized peak power consumption due to all the servers is very low $[10,22]$. Leveraging this fact, they showed significant improvements in the number of applications/servers could be consolidated for the same power supply. Recent work from Raghavendra et al. looked at coordinating multiple power management activities (average and peak) happening throughout the hierarchy of a data center [21]. To the best of our knowledge, we are the first to investigate the prediction of sustained power consumption for a set of consolidated applications. We believe that our prediction techniques will greatly complement all of these other related research efforts. Thermal reliability has extensively been looked at both the server and the data center levels, including techniques to dynamically throttle or move applications upon reliability violations $[3,7,15]$ Recent servers are being shipped with in-built capability to measure power at a very fine time granularity. IBM's Active Energy Manager uses this capability to dynamically measure and control power consumption of a server [16].

Modeling and characterization of power consumption. Modeling of power consumption has been done at various granularities from a data-center, server, individual components to an instruction $[9,14,32]$ either by using direct measurements or estimations from performance counters or a combination of both. SPEC's ongoing effort, SPECPower aims at characterizing the performance and power behavior of servers at different utilizations [25]. To the best of our knowledge, we are the first ones to do such an extensive characterization of power consumption in a consolidated environment, and consider our research complimentary to the work on SPECPower.

\section{Concluding Remarks and Future Work}

Our work was motivated by the need to ensure that emergent techniques for consolidating applications in enterprisescale data centers exhibit robust and predictable power dissipation behavior. Consolidation of workloads has emerged as a key mechanism to dampen the rapidly growing energy expenditure within enterprise-scale data centers. However, before these consolidation-based techniques can be gainfully utilized, we must be able to predict and enforce appropriate limits on power consumption at various levels within the 
data center. In particular, two kinds of power budgetsaverage budgets defined over relatively coarse time-scales and sustained budgets defined over short time-scales-were found to be crucial to the safe and profitable operation of data centers. We used insights gained from detailed profiling of several applications - both individual and consolidated - to develop predictive models for average and sustained power consumption of our server. We implemented our techniques on a Xen-based platform and evaluated them in a wide variety of consolidation settings. Our results were promising. For a variety of consolidation scenarios, we were able to predict average power consumptions with a 5\% error-margin. Our sustained power prediction techniques predict close yet safe upper bounds on the sustained power consumption of consolidated applications.

\section{References}

[1] M. Annavaram, E. Grochowski, and J. Shen. Mitigating Amdahl's Law through EPI Throttling. In Proceedings of the International Symposium on Computer Architecture (ISCA), 2005.

[2] P. Barham, B. Dragovic, K. Fraser, S. Hand, T. Harris, A. Ho, R. Neugebauer, I. Pratt, and A. Warfield. Xen and the art of virtualization. In SOSP '03: Proceedings of the nineteenth ACM symposium on Operating systems principles, pages 164-177, New York, NY, USA, 2003. ACM.

[3] F. Bellosa, S. Kellner, M. Waitz, and A. Weissel. Event-driven energy accounting for dynamic thermal management. In Proceedings of the Workshop on Compilers and Operating Systems for Low Power (COLP'03), 2003.

[4] R. Bianchini and R. Rajamony. Power and Energy Management for Server Systems. IEEE Computer, 37(11), November 2004

[5] P. Bohrer, D. Cohn, E. Elnozahy, T. Keller, M. Kistler, C. Lefurgy, R. Rajamony, F. Rawson, and E. V. Hensbergen. Energy Conservation for Servers. In Proceedings of the IEEE Workshop on Power Management for Real-Time and Embedded Systems, May 2001.

[6] J. S. Chase, D. C. Anderson, P. N. Thakar, A. M. Vahdat, and R. P. Doyle. Managing energy and server resources in hosting centers. In SOSP '01: Proceedings of the eighteenth ACM symposium on Operating systems principles, 2001.

[7] J. Choi, Y Kim, A Sivasubramaniam, J. Srebric, Q Wang, and J. Lee. Modeling and Managing Thermal Proles of Rack-mounted Servers with ThermoStat. In IEEE 13th International Symposium on High Performance Computer Architecture, 2007.

[8] Dell Computers: PowerEdge servers SC1425 Spec Sheet. Dec 2005. www.dell.com/downloads/global/products/pedge/ en/sc1425\_specs.pdf.

[9] D. Economou, S. Rivoire, C. Kozyrakis, and P. Ranganathan. Full-system Power Analysis and Modeling for Server Environments. In Proceedings of the Workshop on Modeling, Benchmarking, and Simulation (MoBS), June 2006.

[10] X. Fan, W.-D. Weber, and L. A. Barroso. Power provisioning for a warehousesized computer. In ISCA '07: Proceedings of the 34th annual international symposium on Computer architecture, 2007.

[11] W. Felter, K. Rajamani, T. Keller, and C. Rusu. A Performance-Conserving Approach for Reducing Peak Power Consumption in Server Systems. In Proceedings of the International Conference on Supercomputing (ICS), June 2005.

[12] M. E. Femal and V. W. Freeh. Safe overprovisioning: Using power limits to increase aggregate throughput. In Workshop on Power-Aware Computer Systems (PACS'04), 2004.

[13] J. Flinn and M. Satyanarayanan. Managing battery lifetime with energy-aware adaptation. ACM Trans. Comput. Syst., 22(2):137-179, 2004.
[14] S. Gurumurthi, A Sivasubramaniam, M. Irwin, N. Vijaykrishnan, M. Kandemir, T. Li, and L. John. Using Complete Machine Simulation for Software Power Estimation: The SoftWatt Approach. In Proceedings of the International Symposium on High Performance Computer Architecture, February 2002 .

[15] T. Heath, A. P. Centeno, P. George, Y. Jaluria, and R. Bianchini. Mercury and Freon: Temperature Emulation and Management in Server Systems. In Proceedings of the International Conference on Architectural Support for Programming Languages and Operating Systems (ASPLOS), October 2006.

[16] IBM Active Energy Manager - Measure and Cap Energy. http : / / www- 03 . ibm.com/press/us/en/pressrelease/22551.wss.

[17] C. Lefurgy, X. Wang, and M. Ware. Server-level power control. In ICAC '07: Proceedings of the Fourth International Conference on Autonomic Computing, page 4, Washington, DC, USA, 2007. IEEE Computer Society.

[18] A. Merkel and F. Bellosa. Balancing Power Consumption in Multiprocessor Systems. In Proceedings of the ACM SIGOPS EuroSys Conference, April 2006.

[19] R. Nathuji and K. Schwan. Virtualpower: Coordinated power management in virtualized enterprise systems. In 21 st ACM Symposium on Operating Systems Principles (SOSP'07), 2007.

[20] E. Pinheiro, R. Bianchini, E. Carrera, and T. Heath. Load Balancing and Unbalancing for Power and Performance in Cluster-Based Systems. In Proceeding of the Workshop on Compilers and Operating Systems for Low Power, Sept 2001.

[21] R. Raghavendra, P. Ranganathan, V. Talwar, Z. Wang, and X. Zhu. No Power Struggles: Coordinated multi-level power management for the data center. In Thirteenth International Conference on Architectural Support for Programming Languages and Operating Systems (ASPLOS '08), Mar. 2008.

[22] P. Ranganathan, P. Leech, D. Irwin, and J. Chase. Ensemble-level Power Management for Dense Blade Servers. In Proceedings of the International Symposium on Computer Architecture (ISCA), June 2006.

[23] Signametrics Multimeter SM2040 Series Spec Sheet. May 2000. http:// www.signametrics.com/products/sm2040.pdf.

[24] SPEC CPU2000. http://www.spec.org/cpu2000/.

[25] SPECpower. http://www.spec.org/specpower/.

[26] M. Steinder, I. Whalley, J. E. Hanson, and J. O. Kephart. Coordinated management of power usage and runtime performance. In Network Operations and Management Symposium (NOMS'08), 2008.

[27] J. Stoess, C. Lang, and F. Bellosa. Energy management for hypervisor-based virtual machines. In Proceedings of the 2007 USENIX Technical Conference (USENIX'07), June 2007.

[28] TPC-W. www.tpc.org/tpcw.

[29] B. Urgaonkar, G. Pacifici, P. Shenoy, M. Spreitzer, and A. Tantawi. An Analytical Model for Multi-tier Internet Services and its Applications. In SIGMETRICS 2005, June 2005.

[30] B. Urgaonkar, P. Shenoy, and T. Roscoe. Resource Overbooking and Application Profiling in Shared Hosting Platforms. In Proceedings of the Fifth USENIX Symposium on Operating Systems Design and Implementation (OSDI 2002), Boston, MA, December 2002.

[31] X. Wang and M. Chen. Cluster-level feedback power control for performance optimization. In Proceedings of the Fourteenth International Symposium on High-Performance Computer Architecture (HPCA'08), Feb. 2008.

[32] W. Wu, L. Jin, J. Yang, P. Liu, and S. X.-D. Tan. Efficient power modeling and software thermal sensing for runtime temperature monitoring. ACM Trans. Des. Autom. Electron. Syst., 12(3):26, 2007. 